\title{
INFLUÊNCIA DE VARIAÇÕES DE MODELO ESTRUTURAL E VIDA ÚTIL DE PROJETO NOS IMPACTOS AMBIENTAIS GERADOS POR EDIFÍCIOS DE CONCRETO ARMADO
}

\author{
CASSINELLI, ANDREMIR 1. \\ Graduando Engenharia Civil \\ UFRGS \\ RS, Brasil \\ andremirlc10@gmail.com
}

GARCEZ, MÔNICA R.

Dra. Engenharia Civil

UFRGS

RS, Brasil

monica.garcez@ufrgs.br

\author{
GODOY, LUIS G. G. \\ MSc. Engenharia Civil \\ UFRGS \\ RS, Brasil \\ luisgodoy.eng@gmail.com \\ SCHWETZ, PAULETE $F$. \\ Dr. Engenharia Civil \\ UFRGS \\ RS, Brasil \\ pauletefs@gmail.com
}

\section{RESUMO}

Em tempos em que o uso consciente de materias é prioridade em muitas áreas da produção de bens e seviços, a engenharia civil está ainda aquém do seu potencial, visto que a atividade gera altos impactos ambientais, principalmente relacionados aos níveis de emissões de poluentes. Entretanto, por meio do uso de técnicas construtivas e estratégias de projeto, podese amenizar o impacto ambiental gerado pelas edificações. $\mathrm{O}$ uso de diferentes sistemas de lajes, por exemplo, pode reduzir o consumo de materiais em edifícios de concreto armado. Por outro lado, há também a possibilidade de aumentar a vida útil de projeto dos edifícios de concreto armado. Desta forma, este estudo propõe uma avaliação da influência de variações de modelo estrutural e vida útil de projeto nos impactos ambientais gerados por edifícios de concreto armado. Os impactos ambientais das diferentes estratégias de projeto estudadas são avaliados pelos resultados de uma Análise de Ciclo de Vida, considerando a produção e transporte dos materiais, a construção da estrutura, e, finalmente o desmonte e descarte, que corresponde ao final do ciclo de vida da estrutura. Os resultados encontrados contribuem para a consolidação de abordagens contemporâneas que sugerem a necessidade de incorporar o grau de impactos ambientais nos projetos estruturais de edifícios de concreto armado.

Palavras-chave: emissão $\mathrm{CO}_{2}$, modelos estruturais, vida útil, ciclo de vida, concreto armado.

\section{ABSTRACT}

In times in which the conscious use of materials is a priority in a lot of areas of goods production and services, civil engineering still is below it's potential, since this activity creates high environmental impacts, mostly related to pollutant emission levels. However, by using constructive techniques and design strategies, the environmental impact generated by buildings can be mitigated. Using different slab systems, for example, can reduce material consumption in reinforced concrete buildings. On the other hand, there is also the possibility of increasing the life cycle of reinforced concrete buildings. Thus, this study proposes an evaluation of the influence of structural model variations and design life cycle on the environmental impacts generated by reinforced concrete buildings. The environmental impacts of different design strategies studied area evaluated by the results of a Life Cycle Analysis, considering the production and transportation of materials, the construction of the structure, and finally the disassembly and final disposal, which corresponds to the end of the life cycle of the structure. These results contribute to the consolidation of contemporary approaches that suggest the need to incorporate the degree of environmental impacts in the structural projects of reinforced concrete buildings. Keywords: $\mathrm{CO}_{2}$ emissions, structural models, life cycle, reinforced concrete.

\section{INTRODUÇÃO}

A energia usada por edificações corresponde a 30-40\% do total da energia primária usada mundialmente [1]. O setor de construção é também um grande responsável pelas emissões dos gases $\mathrm{CO}_{2}$, sendo responsável por $19 \%$ da energia associada às emissões de gases do efeito estufa [2]. Tendo em vista este cenário de grande uso de energia e recursos naturais,se faz necesário estudar métodos e modelos de projeto que tentem minimizar uso de energia e diminuir os impactos ambientais associados às edificações. Os materiais mais usados no setor da construção são o concreto e o aço. Ambos materiais apresentam altos custos energéticos para sua produção, transporte e disposição final [3]. Entretanto, por meio do uso de técnicas construtivas e estratégias de projeto, pode-se amenizar o impacto ambiental gerado pela 
construção de edificações. O uso de diferentes sistemas de lajes, por exemplo, pode reduzir o consumo de materiais em edifícios de concreto armado. Por outro lado, há também a possibilidade de aumentar a vida útil de projeto dos edifícios de concreto armado, a qual gera mais impacto no momento da construção, mas futuramente esse impacto pode ser compensado pelo maior tempo de utilização.

Desta forma, este estudo propõe uma avaliação da influência de variações de modelo estrutural e vida útil de projeto nos impactos ambientais gerados pela construção da estrutura de concreto armado de um edifício. Foram projetados edifícios de concreto armado com dois diferentes sistemas de lajes (maciça e nervurada) e diferentes cobrimentos (35mm e $45 \mathrm{~mm}$ ) para atender vidas úteis de projeto de 50 e 100 anos. Os impactos ambientais das diferentes estratégias de projeto estudadas são avaliados pelos resultados de uma Análise de Ciclo de Vida, considerando a produção e transporte dos materiais, a construção da estrutura, e, finalmente o desmonte e descarte, que corresponde ao final do ciclo de vida da estrutura.

\section{METODOLOGIA}

Nesse estudo foram criados 4 modelos estruturais para uma edificação de concreto armado com 4 pavimentos, em planta de $20 x 30 \mathrm{~m}\left(600 \mathrm{~m}^{2}\right.$ por pavimento), como ilustra a Figura 1. O concreto usado em todos os modelos possui resistência característica à compressão de 50MPa. Os 4 modelos apresentam as seguintes configurações:

- Modelo I: lajes maciças, pilares de 33x33cm e vigas 20x70cm. Lajes com espessura 18,3cm (altura equivalente de laje nervurada para parâmetro comparativo). Cobrimento de $35 \mathrm{~mm}$, atendendo os parâmetro da norma brasileira NBR 6128 [ref], para uma vida útil de 50 anos;

- Modelo II: lajes nervuradas, pilares de 33x33cm e vigas $20 \times 70 \mathrm{~cm}$. Lajes com espessura 28,5cm, forma Atex $65021 \mathrm{~cm}$ nervura, $7,5 \mathrm{~cm}$ mesa. ). Cobrimento de $35 \mathrm{~mm}$, atendendo os parâmetro da norma brasileira NBR 6128[ref], para uma vida útil de 50 anos;

- Modelo III: lajes maciças, pilares de $35 \times 35 \mathrm{~cm}$ e vigas $22 \times 72 \mathrm{~cm}$. Lajes com espessura $19,9 \mathrm{~cm}$ (altura equivalente de laje nervurada para parâmetro comparativo). Cobrimento de $45 \mathrm{~mm}$, atendendo os parâmetro da norma britânica BS 8500-1[ref], para uma vida útil de 100 anos;

- Modelo IV: lajes nervuradas, pilares de 35x35cm e vigas 22x72cm. Lajes com espessura $31 \mathrm{~cm}$, forma Atex 650 $21 \mathrm{~cm}$ nervura, $10 \mathrm{~cm}$ mesa. Cobrimento de $45 \mathrm{~mm}$, atendendo os parâmetro da norma britânica BS 8500-1[ref], para uma vida útil de 100 anos;

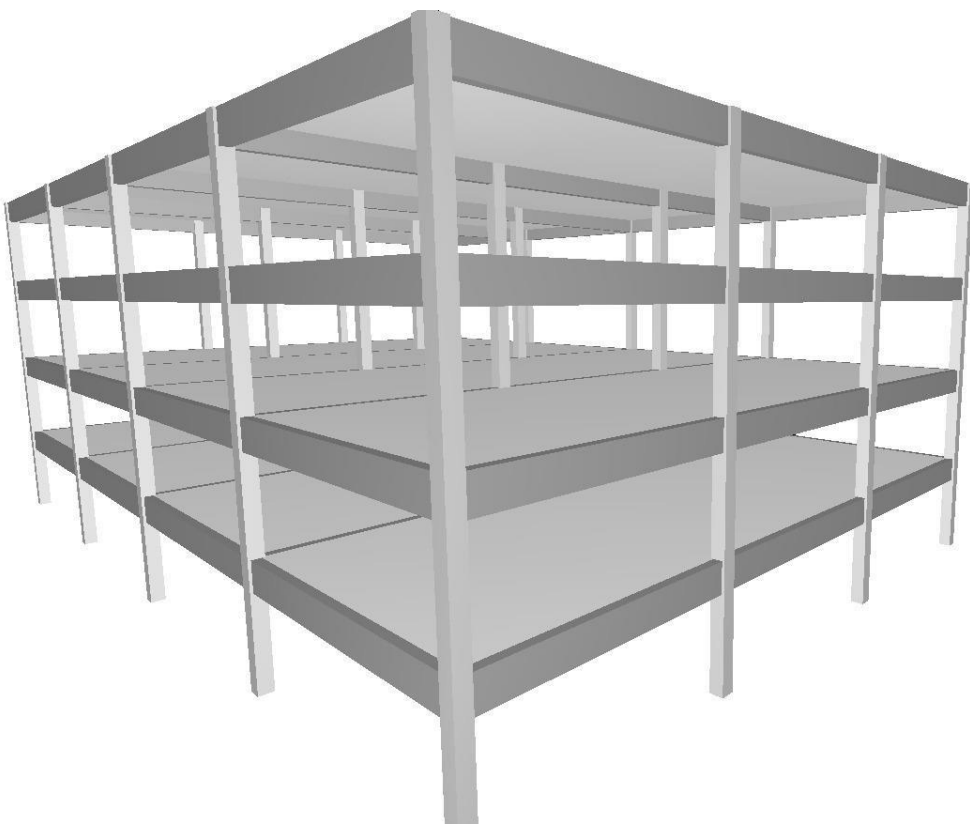

Figura 1: Modelo 3D da edificação modelo.

Os carregamentos usados, além do peso próprio dos elementos estruturais, foram de $13 \mathrm{KN} / \mathrm{m}$ de peso próprio para as vigas externas (carregamento de paredes de alvenaria), $3 \mathrm{KN} / \mathrm{m}^{2}$ de carga variável e $0,85 \mathrm{KN} / \mathrm{m}^{2}$ como carga do resvestimento dos pisos. 
Os modelos estruturais foram simulados no software de cálculo estrutural TQS [4], utilizando o modelo estrutural IV do software, o qual utiliza um modelo de vigas e pilares flexibilizado. Esse modelo é feito em pórtico espacial com um sistema de grelhas para os pavimentos. O pórtico é composto por barras que simulam as vigas e pilares da estrutura. Nas lajes somente os efeitos verticais são considerados. A estrutura foi considerada estável até que o software não apresentasse erros graves nos resultados. Com a simulação estrutural pronta, foram extraídos os quantitativos dos materiais que foram usados para as análises dos impactos ambientais. As fundações não foram consideradas nesse estudo, visto que essa parte da estrutura é muito varíavel de acordo com a região e tipo de solo em que a edificação se encontra, logo para que os critérios de comparação fossem mais genéricos, optou-se por não incluir os impactos gerados por fundações.

Conforme citado anteriormente, o concreto usado na edificação apresenta resistência de 50MPa. Foi considerado o traço apresentado na Tabela 1 para a obtenção da quantidade de materiais, seguindo o método proposto pelo ACI 211.1 [5]. O traço se refere ao cimento Portland tipo CPII - F ( com 6-10\% de massa calcária, agregado graúdo de basalto $\left(2.90 \mathrm{~g} / \mathrm{cm}^{3}\right.$, Dmax 25mm), areia (2.63 $\mathrm{g} / \mathrm{cm}^{3}$, finura 2.4$)$, aditivo plastificante e 1,5\% de ar aprisionado [6].

Tabela 1 - Traço do concreto utilizado

\begin{tabular}{llllll}
\hline Concreto & $\begin{array}{l}\text { Cimento } \\
\mathbf{k g} / \mathbf{m}^{\mathbf{3}}\end{array}$ & $\begin{array}{l}\text { Areia } \\
\mathbf{k g} / \mathbf{m}^{\mathbf{3}}\end{array}$ & $\begin{array}{l}\text { Brita } \\
\mathbf{k g} / \mathbf{m}^{\mathbf{3}}\end{array}$ & $\mathbf{A} / \mathbf{C}$ & $\begin{array}{l}\text { Aditivo } \\
\mathbf{1 / \mathbf { m } ^ { 3 }}\end{array}$ \\
\hline $\mathrm{C} 50$ & 500 & 644,24 & 1192,8 & 0,34 & 3 \\
\hline
\end{tabular}

$\mathrm{Na}$ análise de impacto ambiental, foi utilizado o método LCA (Avaliação do Ciclo de ida), baseado nos padrões internacionais ISO 14040 [7] e ISO 14044 [8]. Utilizou-se o software de avaliação de impactos open source OpenLCA [9]. Esse software utiliza a metodologia LCA, que leva em conta todos os aspectos de uso de recursos e disposição no meio, associado a um sistema industrial desde a fabricação de determinado produto até o fim de sua vida útil. O método LCA considera desde a extração de matéria-prima, produção, distribuição e disposição final de um produto. Seu objetivo é ser uma ferramenta de comparação entre alternativas envolvidas em todas as fases que um produto passa [10].

A unidade funcional considerada é o sistema estrutural de uma edificação comercial de 4 pavimentos formado por pilares e vigas em concreto armado e lajes maciças e nervuradas para cada modelo. Nesse trabalho foram considerados as etapas envolvidas na produção de materiais, a qual corresponde à fase de produto da BS 15978 [11], incluindo fornecimento de matéria-prima (A1), transporte (A2), e produção (A3) de concreto, aço e formas. A construção da estrutura de concreto armado corresponde à fase de construção da BS 15978 [11], que inclui transporte (A4) e o processo de instalação/construção (A5). Final de vida de projeto inclui demolição (C1), transporte (C2), separação dos resíduos (C3) e disposição final (C4) de acordo com a fase de final de vida da BS 15978 [11]. Estão fora do escopo do trabalho a contabilização dos impactos referentes a atividades de manutenção durante a vida útil das estruturas e possíveis modificações futuras nas edificações, como reformas para modernização. A Figura 2 apresenta esquematicamente as fronteiras do sistema analisado.

As distâncias de transporte consideradas nesse estudo, mostradas na Tabela 2, referem-se a um cenário de construção em uma cidade de tamanho médio (496,8 km², 1,5 milhões de habitantes) no sul do Brasil [12].

Tabela 2 - Distâncias de transporte consideradas nesse estudo

\begin{tabular}{lrr}
\hline Fluxo de Entrada & Modo de transporte & Distância (km) \\
\hline Transporte do aditivo da fábrica até a concreteira & Caminhão (27 ton.) & 1100 \\
Transporte do cimento da fábrica até a concreteira & Trem & 950 \\
Transporte de agregados graúdos da fábrica até a concreteira & Caminhão (27 ton.) & 60 \\
Transporte da areia até a concreteira & Barca & 100 \\
Transporte do concreto da concreteira para o canteiro & Caminhão (27 ton.) & 20 \\
Tranporte do aço da fábrica para o canteiro & Caminhão (27 ton.) & 30 \\
Transporte das formas de madeira para o canteiro & Caminhão (27 ton.) & 750 \\
Transporte das formas plásticas até o canteiro & Caminhão (27 ton.) & 1250 \\
Disposição final para o aterro & Caminhão (27 ton.) & 30 \\
\hline
\end{tabular}


Os processos e fluxos considerados na avaliação dos impactos ambientais, bem como os dados obtidos por meio da análise estrutural, estão apresentados na Tabela 3, levando em consideração quantidades de materiais, energia usada na produção dos materiais, construção da estrutura de concreto armado e o final de vida. Na análise de impacto que o software OpenLCA, o produto da distância pela massa de material transportado é levado em consideração como medida do impacto causado pelo transporte, estando esses valores apresentados na Tabela 4.

Tabela 3 - Processos e fluxos considerados na avaliação dos impactos ambientais.

\begin{tabular}{|c|c|c|c|c|c|c|c|}
\hline Processo & Fluxo de Saída & Fluxo de Entrada & $\begin{array}{r}\text { Modelo } \\
\text { I }\end{array}$ & $\begin{array}{r}\text { Modelo } \\
\text { II }\end{array}$ & $\begin{array}{r}\text { Modelo } \\
\text { III }\end{array}$ & $\begin{array}{r}\text { Modelo } \\
\text { IV }\end{array}$ & Unidade \\
\hline \multirow{13}{*}{$\begin{array}{l}\text { Produção } \\
\text { de } \\
\text { materiais }\end{array}$} & \multirow{10}{*}{ Concreto } & Aditivo & 1548,6 & 1202,4 & 1692,0 & 1403,1 & $\overline{1}$ \\
\hline & & Transporte do aditivo da fábrica até a concreteira & 1,9 & 1,5 & 2,1 & 1,7 & $\mathrm{t}$ \\
\hline & & Água & 87,8 & 68,1 & 95,9 & 79,5 & $\mathrm{t}$ \\
\hline & & Cimento & 258,1 & 200,4 & 282,0 & 233,9 & $\mathrm{t}$ \\
\hline & & Transporte do cimento da fábrica até a concreteira & 258,1 & 200,4 & 282,0 & 233,9 & $\mathrm{t}$ \\
\hline & & Agregado graúdo & 615,7 & 478,1 & 672,7 & 557,9 & $\mathrm{t}$ \\
\hline & & $\begin{array}{l}\text { Transporte de agregado graúdo da fábrica até } \\
\text { a concreteira }\end{array}$ & 615,7 & 478,1 & 672,7 & 557,9 & $\mathrm{t}$ \\
\hline & & Areia & 332,6 & 258,2 & 363,4 & 301,3 & $\mathrm{t}$ \\
\hline & & Transporte da areia até a concreteira & 332,6 & 258,2 & 363,4 & 301,3 & $\mathrm{t}$ \\
\hline & & Energia para preparo do concreto & 1651,8 & 1282,6 & 1804,8 & 1496,6 & $\mathrm{kWh}$ \\
\hline & \multirow{2}{*}{ Formas } & Formas de madeira & 3343,0 & 1037,8 & 3373,9 & 1070,1 & $\mathrm{~m}^{2}$ \\
\hline & & Formas plásticas & - & 742,4 & - & 878,4 & $\mathrm{~kg}$ \\
\hline & Aço & Aço & 42,2 & 24,2 & 46,7 & 26,9 & $\mathrm{t}$ \\
\hline \multirow{7}{*}{$\begin{array}{l}\text { Construção } \\
\text { da } \\
\text { estrutura } \\
\text { de concreto } \\
\text { armado }\end{array}$} & Transporte dos & Transporte do concreto da concreteira para o canteiro & 516,2 & 400,8 & 564,0 & 467,7 & $\mathrm{~m}^{3}$ \\
\hline & materiais para & Transporte das formas de madeira até o canteiro & 27,8 & 8,6 & 28,1 & 8,9 & $\mathrm{t}$ \\
\hline & o canteiro de & Transporte das formas plásticas para o canteiro & 0 & 742,4 & 0 & 878,4 & $\mathrm{~kg}$ \\
\hline & obras & Transporte do aço da fábrica para o canteiro & 42,2 & 24,2 & 46,7 & 26,9 & $\mathrm{t}$ \\
\hline & $\begin{array}{l}\text { Água para cura } \\
\text { do concreto }\end{array}$ & Água para cura do concreto & 457798,2 & 457798,2 & 457798,2 & 457798,2 & 1 \\
\hline & $\begin{array}{l}\text { Bombeamento } \\
\text { do concreto }\end{array}$ & Diesel para bombeamento & 2581,0 & 2004,0 & 2820,0 & 2338,5 & $\mathrm{kWh}$ \\
\hline & $\begin{array}{l}\text { Compactação do } \\
\text { concreto }\end{array}$ & Eletricidade para compactação do concreto & 15486,0 & 12024,0 & 16920,0 & 14031,0 & $\mathrm{kWh}$ \\
\hline \multirow{4}{*}{$\begin{array}{l}\text { Fim da } \\
\text { vida útil }\end{array}$} & \multirow{4}{*}{$\begin{array}{l}\text { Demolição e } \\
\text { disposição final }\end{array}$} & Diesel para demolição da estrutura de concreto armado & 171351,7 & 133044,9 & 187218,8 & 155252,2 & $\mathrm{kWh}$ \\
\hline & & Diesel de máquina carregadeira no canteiro & 5313,2 & 4125,4 & 5805,2 & 4814,0 & $\mathrm{kWh}$ \\
\hline & & Transporte para aterro & 1313,7 & 1020,0 & 1435,4 & 1190,3 & $\mathrm{t}$ \\
\hline & & Disposição final do concreto e aço & 1313,7 & 1020,0 & 1435,4 & 1190,3 & $\mathrm{t}$ \\
\hline
\end{tabular}

Tabela 4 - Produto das distâncias de transporte pela massa de material

\begin{tabular}{|c|c|c|c|c|c|}
\hline Fluxo de Entrada & $\begin{array}{c}\text { Modelo } \\
\text { I }\end{array}$ & $\begin{array}{c}\text { Modelo } \\
\text { II }\end{array}$ & $\begin{array}{c}\text { Modelo } \\
\text { III }\end{array}$ & $\begin{array}{c}\text { Modelo } \\
\text { IV }\end{array}$ & Unidade \\
\hline Transporte do aditivo da fábrica até a concreteira & 2064,0 & 1602,6 & 2255,1 & 1870,1 & $\mathrm{t} * \mathrm{~km}$ \\
\hline Transporte do cimento da fábrica até a concreteira & 245195,0 & 190380,0 & 267900,0 & 222157,5 & $\mathrm{t} * \mathrm{~km}$ \\
\hline Transporte de agregados graúdos da fábrica até a concreteira & 36943,4 & 28684,5 & 40364,4 & 33472,4 & $\mathrm{t}^{*} \mathrm{~km}$ \\
\hline Transporte da areia até a concreteira & 33255,7 & 25821,1 & 36335,1 & 30131,1 & $\mathrm{t}^{*} \mathrm{~km}$ \\
\hline Transporte do concreto da concreteira para o canteiro & 10324,0 & 8016,0 & 11280,0 & 9354,0 & $\mathrm{t}^{*} \mathrm{~km}$ \\
\hline Transporte das formas de madeira para o canteiro & 20893,75 & 6486,25 & 21086,88 & 6688,12 & $\mathrm{t} * \mathrm{~km}$ \\
\hline Transporte das formas plásticas até o canteiro & - & 928 & - & 1098 & $\mathrm{t} * \mathrm{~km}$ \\
\hline Tranporte do aço da fábrica para o canteiro & 1265,0 & 726,5 & 1401,6 & 807,2 & $\mathrm{t} * \mathrm{~km}$ \\
\hline Disposição Final & 39411,3 & 30600,6 & 43060,8 & 35708,4 & $\mathrm{t} * \mathrm{~km}$ \\
\hline
\end{tabular}




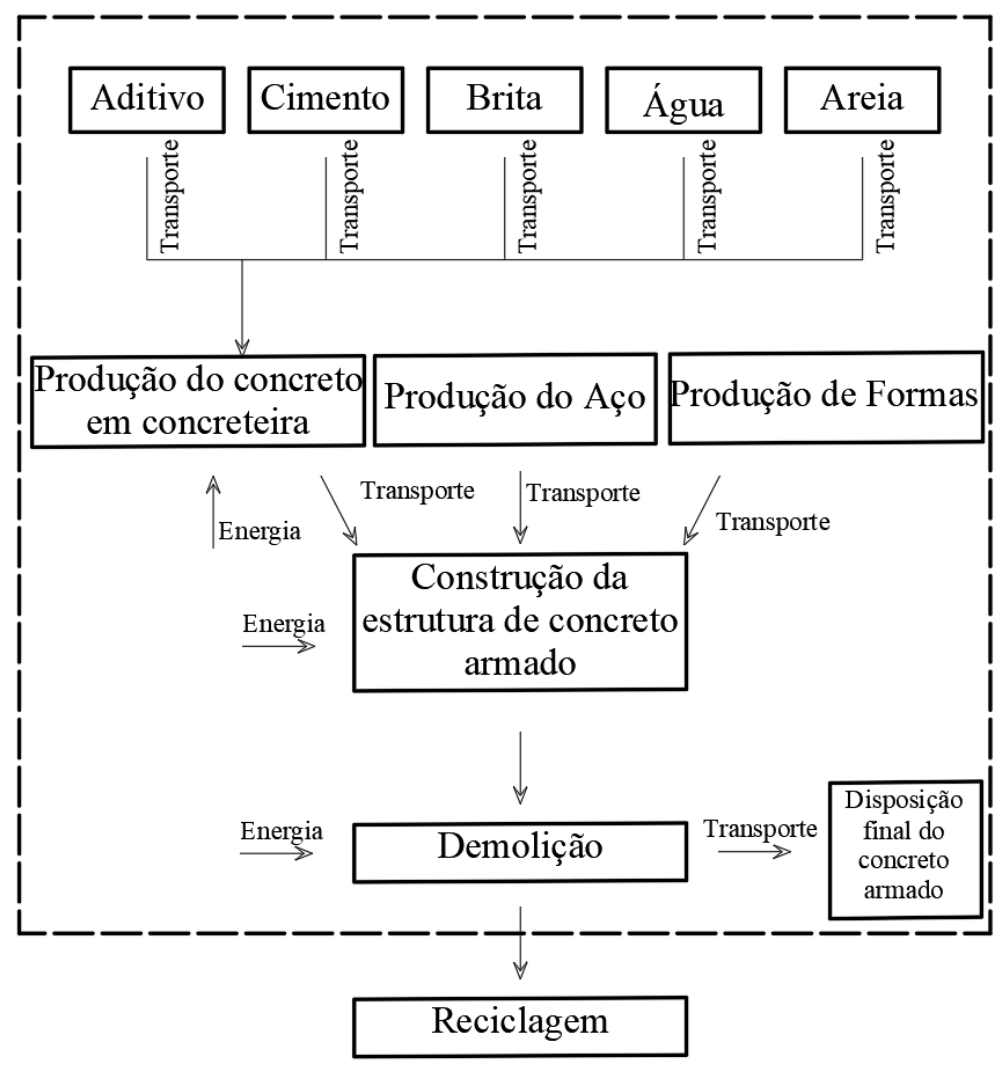

Figura 2: Fronteiras do sistema.

$\mathrm{Na}$ análise foi utilizado o modelo cut-off, que considera que a produção primária de materiais é sempre alocada ao usuário primário do material. Se o material é reciclado, o produtor primário não recebe crédito pelo fornecimento de materiais reciclados [13]. O database utilizado foi o Ecoinvent versão 3.5 [14], utilizando modificações sempre que necessário para adaptar às necessidades e condições brasileiras.

A avaliação de impacto foi baseada no método de pressão orientada CML (baseline), desenvolvido pela Universidade de Leiden em 2001 [15]. Quanto às categorias de impacto analisadas, as seguintes foram escolhidas: potencial de acidificação, mudança climática, potencial de eutrofização, ecotoxicidade aquática de água doce, toxicidade humana, ecotoxicidade aquática marinha, oxidação fotoquímica, depleção estratosférica de ozônio e ecotoxicidade terrestre. [16].

\section{RESULTADOS}

A avaliação de impacto ambiental foi realizada para os 4 modelos. Os impactos ambientais gerados pelos diferentes modelos estão mostrados nas Figuras 3 a 6, a seguir, Na Figura 3 são comparados os modelos estruturais de laje maciça (Modelo I) e laje nervurada (Modelo II), ambos dimensionados para uma vida útil de 50 anos (cobrimento 35mm), sendo os impactos do Modelo I considerados como 100\%. Nota-se que o Modelo II apresenta menor impacto em todas as categorias consideradas, o que é coerente com o maior consumo de concreto, maior peso próprio e consequentemente maior uso de aço nas lajes maciças. Na Figura 4 é feita a mesma comparação, para uma vida útil de 100 anos. Novamente observam-se maiores impactos ambientais gerados pela estrutura com laje maciça, porém com mesmos níveis de depleção estratosférica de ozônio. A depleção estratosférica de ozônio refere-se ao fenômeno de diminuição da densidade de ozônio pelo afinamento da camada de ozônio estratosférico, como resultado de poluentes antropogênicos, resultando no aumento da exposição da pele humana aos raios ultravioleta. Observa-se que a diferença entre os impactos ambientais gerados foi mais pronunciada nos modelos dimensionados para vida útil de projeto de 50 anos. 


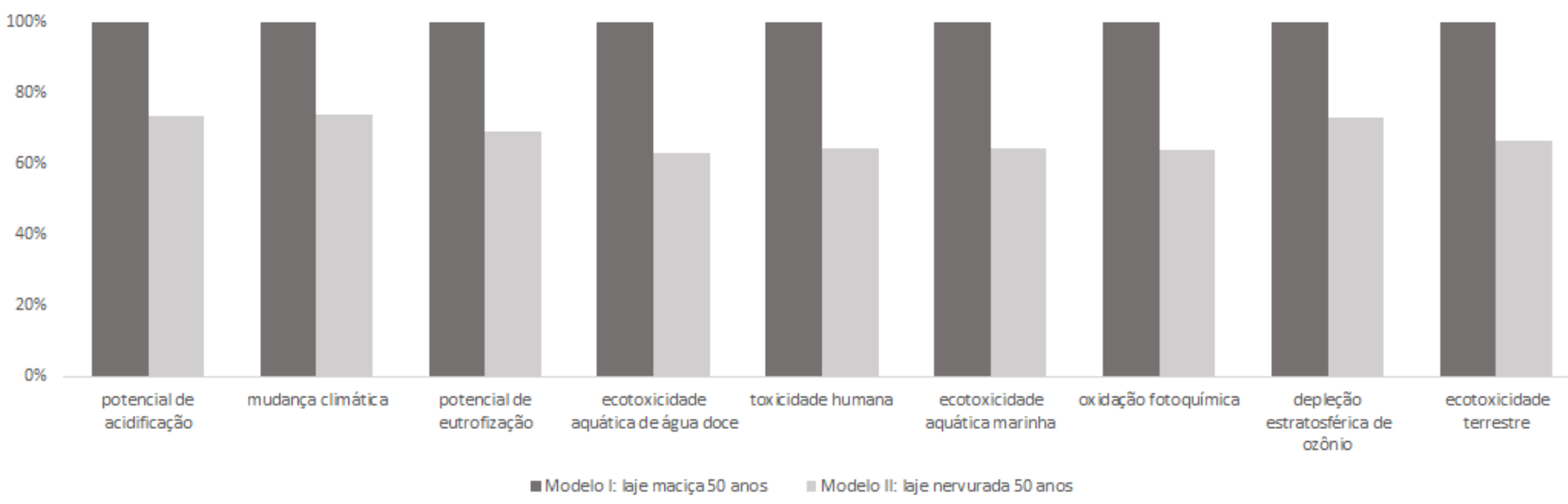

Figura 3: Impactos ambientais para lajes maciça e nervurada e vida útil de 50 anos.

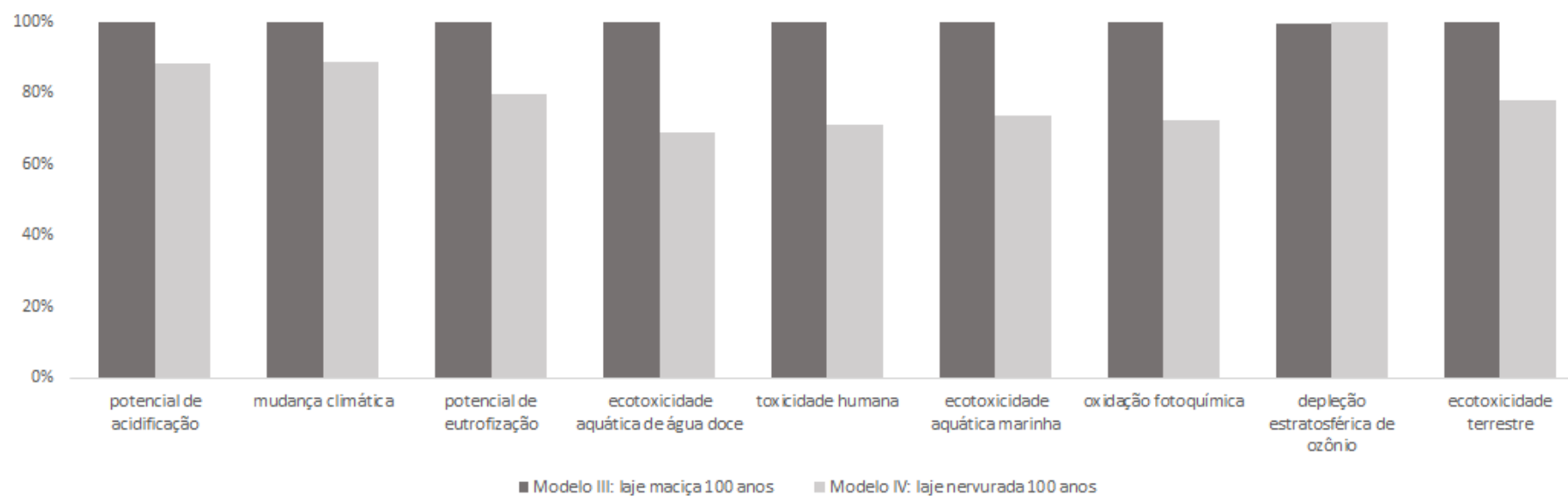

Figura 4: Impactos ambientais para lajes maciça e nervurada e vida útil de 100 anos.

A figura 5 mostra o comparativo da estrutura dimensionada com laje maciça para vidas úteis de projeto de 50 e 100 anos. Nota-se que esta estrutura, quando dimensionada para uma vida útil de projeto de 50 anos, gera cerca de $90 \%$ dos impactos ambientais da estrutura dimensionada para uma vida útil de projeto de 100 anos.

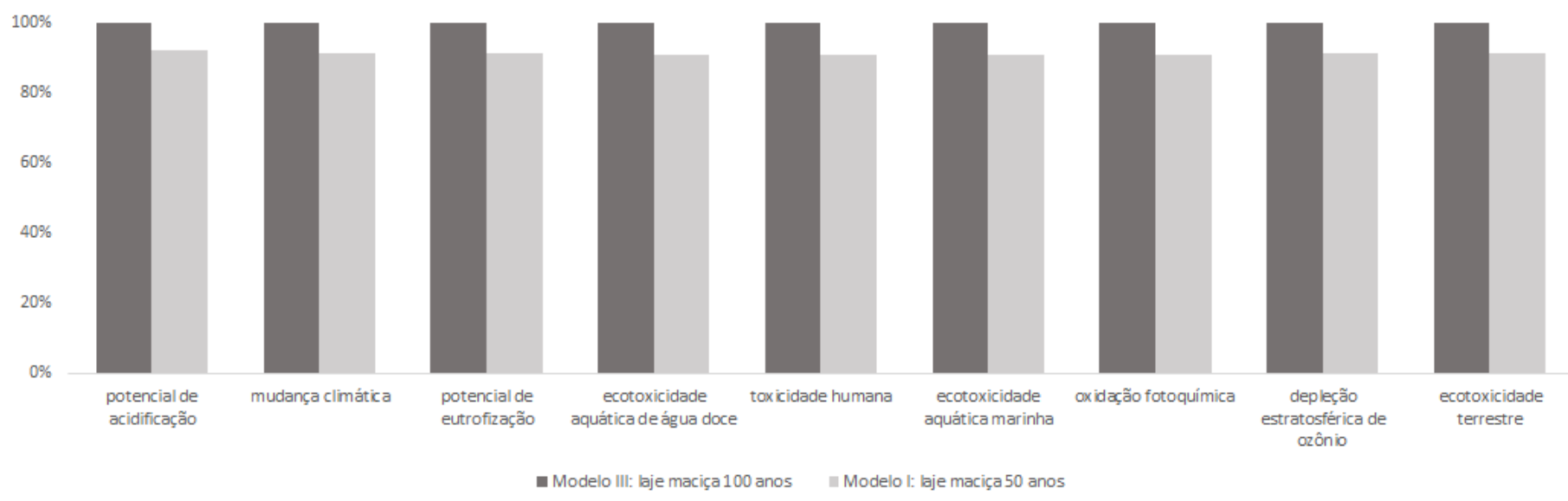

Figura 5: Impactos ambientais para laje maciça considerando vidas uteis de 50 e 100 anos. 
A Figura 6 mostra o comparativo do projeto estrutural dimensionado considerando o sistema estrutural laje nervurada para vidas úteis de 50 e 100 anos. Observa-se a que estrutura projetada com cobrimento de concreto $35 \mathrm{~mm}$, para atender uma vida útil de projeto de 50 anos, gera 65-85\% dos impactos ambientais gerados pela estrutura dimensionada para uma vida útil de projeto de 100 anos.

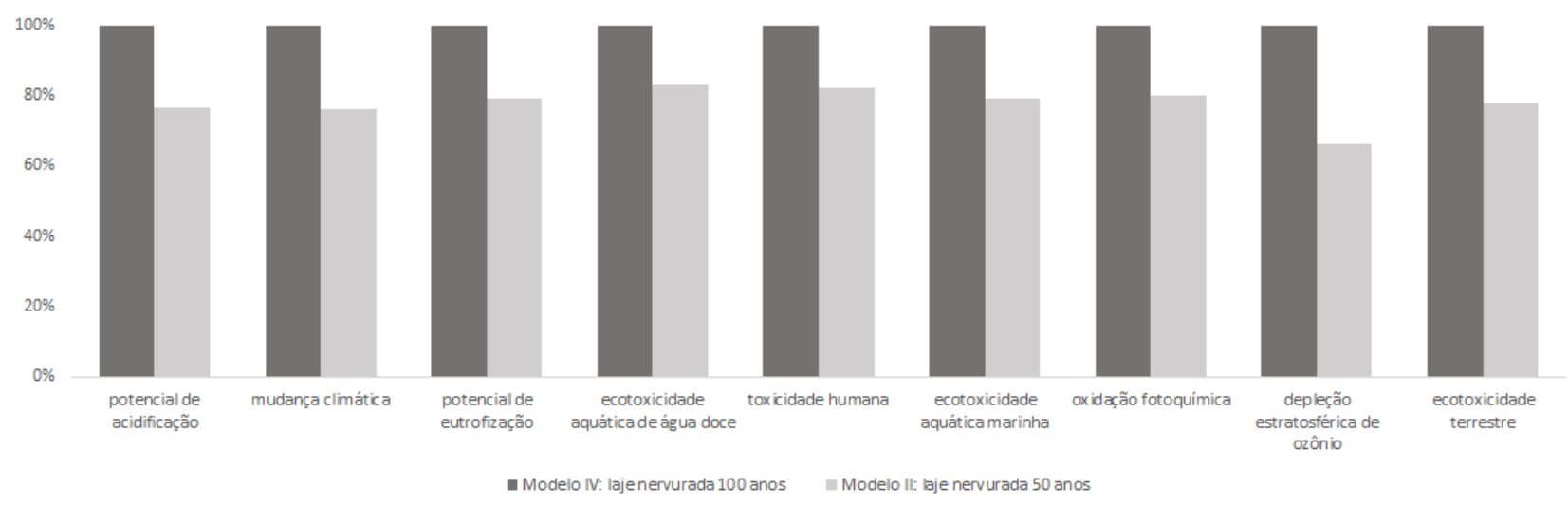

Figura 6: Impactos ambientais para laje nervurada considerando vidas uteis de 50 e 100 anos.

Estes resultados mostram claramente que o aumento do cobrimento do concreto de $35 \mathrm{~mm}$ para $45 \mathrm{~mm}$, para atender os critérios de projeto da BS 8500-1 [ref] para uma vida útil de projeto de 100 anos, mostra vantagens com relação à geração de impactos ambientais. Adicionalmente, é senso comum que a durabilidade de uma estrutura de concreto armado está diretamente relacionada à espessura do concreto que recobre a armadura de aço, sendo espessuras de cobrimento superiores associadas a menor necessidade de intervenções de reparo e manutenção, levando também a uma menor geração de resíduos pela indústria da construção [17].

A Figura 7 mostra os percentuais dos impactos ambientais gerados em cada modelo, pela produção de materiais, construção da estrutura e descarte final da edificação. Nota-se que a fase de produção dos materiais é responsável pela maior geração de impactos ambientais, em todas as categorias de impacto consideradas. De fato, a etapa de produção de materiais está relacionada a grande dispêndio de energia, pois considera desde a extração das matérias primas, até e o transporte para a produção dos materiais. A fase de descarte final é a segunda maior geradora de impactos ambientais, principalmente decorrentes da energia consumida para o desmonte da estrutura, remoção e disposição final dos resíduos.

O modelo III, com laje maciça e 100 anos de vida útil de projeto foi o que mais gerou impacto entre os modelos estudados. O modelo II, com laje maciça, foi o que menos gerou impacto, entretanto ele foi dimensionado para uma vida útil de 50 anos. Apenas considerando os aspectos relacionados aos impactos ambientais, o modelo IV, com laje nervuradas e vida útil de projeto de 100 anos, é o modelo mais atrativo. Em relação aos diferentes tipos de sistemas estruturais, as lajes nervuradas apresentaram um desempenho superior às maciças com relação à geração de menos impactos ambientais, visto que se tem menor consumo de concreto, menor peso próprio e menor consumo de aço, o que implica em menores emissões em toda cadeia produtiva.

Por meio dos dados coletados, é possível afirmar que o projeto de estruturas de concreto armado para uma vida útil de 100 anos é vantajoso, tanto do ponto de vista da durabilidade como do ponto de vista da geração de menores impactos ambientais ao longo do ciclo de vida da estrutura. Nos dois modelos dimensionados para uma durabilidade de 100 anos, o incremento gerado no impacto é pequeno quando se leva em consideração que a edificação foi projetada para ser utilizada pelo dobro do tempo. O modelo estrutural dimensionado com lajes maciças e vida útil de projeto de 100 anos apresentou em média incremento de 9,67\% nos impactos ambientais, com relação ao modelo dimensionado para uma vida útil de projeto de 50 anos. Já no modelo que considerou o sistema de lajes nervuradas, o projeto para vida útil de 100 anos resultou impactos ambientais $28,5 \%$ superiores ao da mesma estrutura dimensionada para uma vida útil de projeto de 50 anos. 

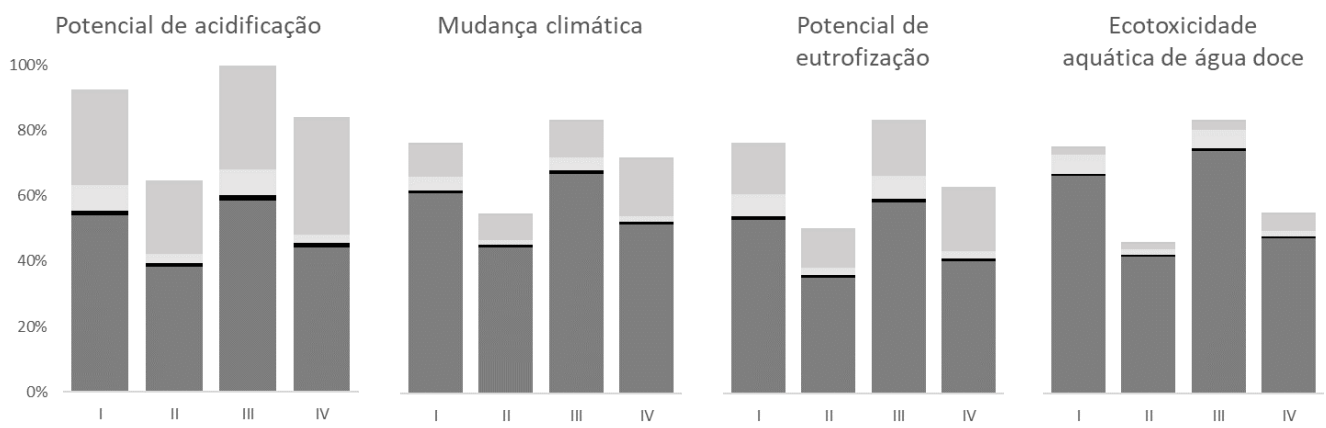

Toxicidade humana aquática de água doce
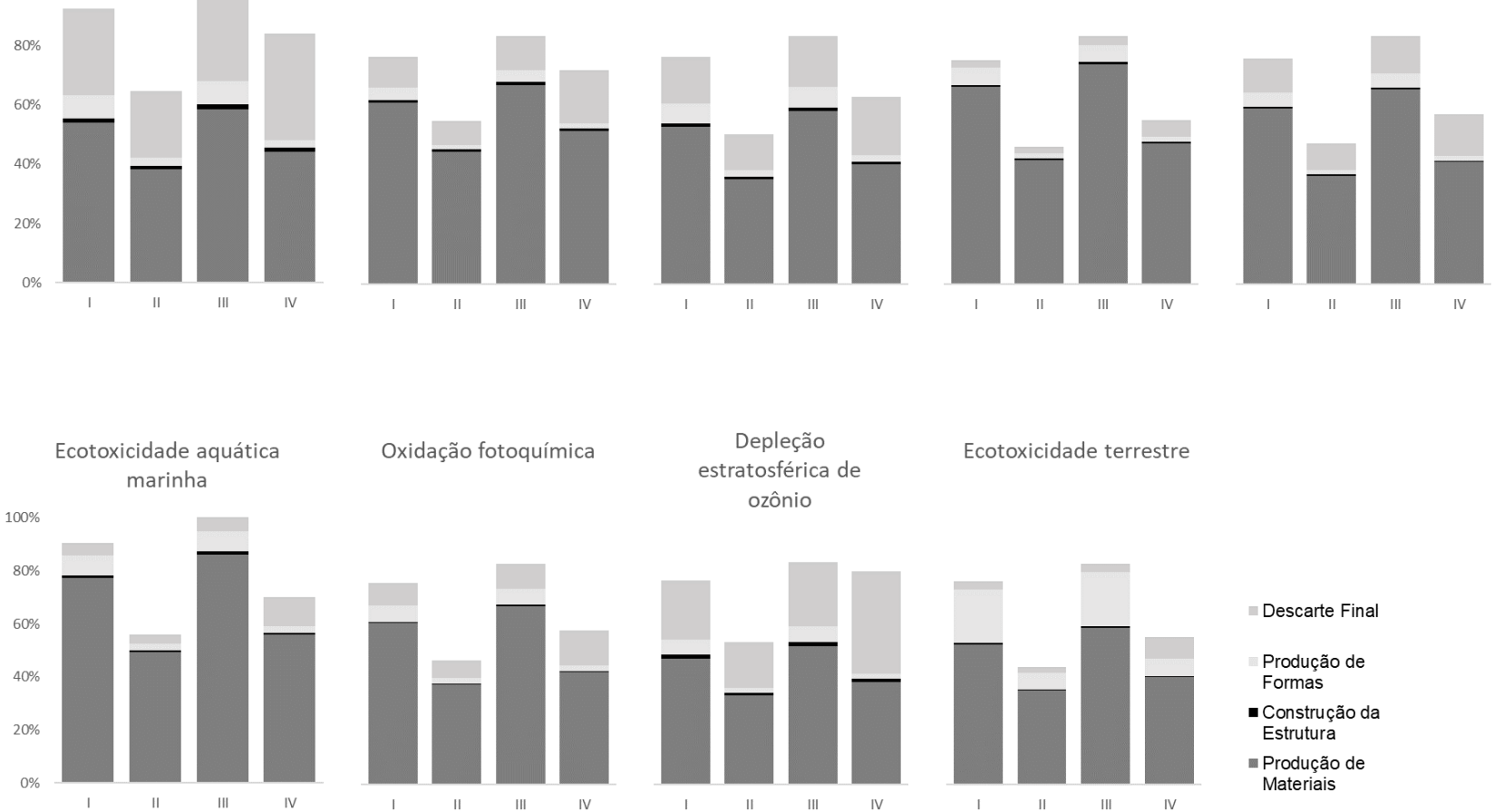

$$
\text { Oxidação fotoquímica }
$$$$
\begin{gathered}
\text { Depleção } \\
\text { estratosférica de } \\
\text { ozônio }
\end{gathered}
$$

Ecotoxicidade terrestre ozônio
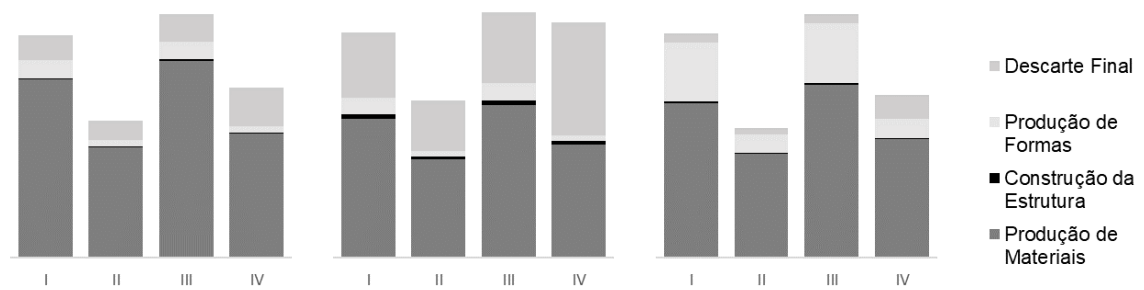

Figura 7: Impactos ambientais gerados pelos 4 modelos estruturais.

\section{CONCLUSÕES}

Esse artigo apresenta um estudo comparativo em um edifício comercial de 4 pavimentos em concreto armado dimensionado considerando dois diferentes sistemas estruturais e vidas úteis de 50 e 100 anos, usando a metodologia LCA, com o objetivo de avaliar o impacto ambiental gerado pelos diferentes modelos.

Os modelos dimensionados com sistema de laje maciça apresentaram maiores impactos ambientais quando comparados com os modelos dimensionados com laje nervurada, independente da vida útil de projeto considerada. Entretanto, esta diferença foi mais pronunciada nos modelos dimensionados para vida útil de projeto de 50 anos. Os modelos dimensionados considerando vida útil de projeto de 100 anos resultaram impactos ambientais 9,67\% (laje maciça) e 28,5\% (laje nervurada) superiores aos modelos dimensionados para uma vida útil de projeto de 50 anos. A fase de produção dos materiais foi a maior responsável pela geração dos impactos ambientais nos 4 modelos estudados, como resultado dos elevados valores de energia incorporada relacionados à produção do cimento e aço, bem como ao transporte das matérias primas.

Os resultados encontrados contribuem para a consolidação de abordagens contemporâneas que sugerem a necessidade de incorporar o grau de impactos ambientais nos projetos estruturais de edifícios de concreto armado.

\section{REFERÊNCIAS}

[1] UNEP-SBCI, Buildings and Climate Change: Status, Challenge and Opportunities, UNEP Publication, Paris, France, 2007

[2] IPCC, Climate Change 2014: Mitigation of Climate Change. Contribution of Working Group III to the Fifth Assessment Report of the Intergovernmental

[3] DI FILIPPO, J; KARPMAN J; DESHAZO J.R. The impacts of policies to reduce CO2 emissions within the concrete supply - Luskin School of Public Affairs, University of California, Los Angeles

[4] http://www1.tqs.com.br/v21 
[5] ACI 211.1, Standard Practice for Selecting Proportions for Normal, Heavyweight, and Mass Concrete, ACI, Farmington Hills, EUA, 1991.

[6] ROHDEN A. B.; GARCEZ M.R. Increasing the sustainability potential of a reinforced concrete building through design strategies: Case study

[7] ISO, International Standardization Organization, 2006. Environmental Management e Life Cycle Assessment Principles and Framework, ISO 14040, Geneve, Switzerland, p. 20.

[8] ISO, International Standardization Organization, 2006. Environmental Management e Life Cycle Assessment e Requirements and Guidelines, ISO 14044, Geneve, Switzerland, p. 46.

[9] OpenLCA, OpenLCA Software See, OpenLCA, Berlin, Germany, 2019.

[10]M.A. Curran Life-Cycle Assessment Encyclopedia of Ecology, Volume 4, 2016, Pages 359-366

[11]BSI, British Standards Institution, BS 15978:2011 Sustainability of Construction Works. Assessment of Environmental Performance of Buildings. Calculation Method, BSI, British Standards Institution, London, UK, 2011. [12]GARCEZ M.R.; ROHDEN A. B.; GODOY L. G. G. D. The role of concrete compressive strength on the service life and life cycle of a RC structure: Case study

[13]https://www.ecoinvent.org/database/system-models-in-ecoinvent-3/cut-off-system-model/allocation-cut-off-byclassification.html

[14]SCLCI, Ecoinvent Database v.3.5. See, SCLCI, Zurich, Switzerland, 2019.

[15]J.B. Guinée, M. Gorrée, R. Heijungs, G. Huppes, R. Kleijn, A. Konin, L. Van Oers, A. Wegener Sleeswijk, S. Suh, H.A. Udo de Haes, H. Bruijn, R. Van Duin, M.A. J. Huijbregts, Handbook on Life Cycle Assessment. Operational Guide to the ISO Standards, Kluwer Academic Publishers, Dordrecht, Netherlands, 2002.

[16]R. Frischknecht, F. Wyss, S.B. Knöpfel, T. Lützkendorf, M. Balouktsi, Cumulative energy demand in LCA: the energy harvested approach, Int. J. Life Cycle Assess. 20 (7) (2015) 957-969.

[17]DA SILVA, M. R.; ROHDEN, A. B.; MESQUITA, E. F. T.; GARCEZ, M. R.; Influence of concrete cover in the service life of a newly built reinforced concrete structure: a case study 